PIPELINE ${ }^{\text {Th }}$ FLEX

EMBOLIZATION DEVICE

WITH SHIELD TECHNOLOGY ${ }^{m}$

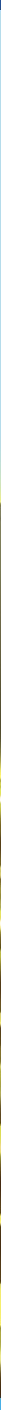

FULL FLOW DIVERSION EFFECT, 


\title{
Diagnostic performance of reformatted isotropic thin-section helical CT images in the detection of superior semicircular canal dehiscence
}

\author{
Gianvincenzo Sparacia ${ }^{1}$ and Alberto laia ${ }^{2}$
}

\begin{abstract}
Purpose: The purpose of this article is to assess the diagnostic performance of computed tomography (CT) reformatted images for detection of superior semicircular canal (SSC) dehiscence.

Material and methods: Forty-two patients, with sound- and/or pressure-induced vestibular symptoms, and 42 control participants underwent helical CT examination with a highly collimated beam $(0.5 \mathrm{~mm})$.

Reformatted images of the vestibular labyrinth were obtained in the standard axial and coronal planes (group A images), and in a plane parallel and perpendicular to the SSC (group B images). Diagnostic performance obtained by evaluating the group A images alone and the group B images alone was analyzed by using the area under the receiver operating characteristic curve (AUC).

Results: The diagnostic performance of group A images was AUC $=0.929$ with an overall accuracy of $92.9 \%$. The diagnostic performance of group B images was AUC $=0.988$ with an overall accuracy of $98.8 \%$. The evaluation of group B images alone showed an improved diagnostic performance over the group A images alone.

Conclusion: Thin-section $0.5-\mathrm{mm}$ collimation CT with reformatted images oriented in the plane parallel and perpendicular to the SSC improves diagnostic accuracy in assessing for SSC dehiscence in comparison to CT images with reconstructions limited to traditional axial and coronal planes.
\end{abstract}

\section{Keywords}

CT, computed tomography, temporal bone, Tullio phenomenon, superior semicircular canal, dehiscence of the superior semicircular canal

\section{Introduction}

Superior semicircular canal (SSC) dehiscence syndrome, first described by Minor et al., ${ }^{1}$ is a pathology that implies vestibular and cochlear symptoms resulting from the communication of the SSC and the adjacent middle cranial fossa.

The symptomatology can be characterized by the presence of dizziness, autophony and hearing loss; vertigo may be induced and aggravated by loud noises (Tullio phenomenon $)^{2}$ and/or pressure variations secondary to intense efforts or in the setting of the Valsalva maneuver (Hennebert's sign). ${ }^{3}$

The dehiscence of the SSC leads to the "third mobile window" (in addition to the oval and round windows), in which the mechanical stimulations (sound and/or pressure) that activate the cochlea may induce endolymphatic flow within the superior semicircular canal and the vestibular labyrinth, leading to dizziness. ${ }^{4,5}$

Potential etiologies for SSC dehiscence include abnormal development of the ducts, congenital defects, chronic otitis, cholesteatoma, and fibrous dysplasia. ${ }^{1,4,5}$
Physical examination signs to diagnose SSC dehiscence syndrome include vertical-torsional eye movements that align with the plane of the SSC evoked by sound and/or pressure stimuli. ${ }^{6-8}$

Computed tomography (CT) imaging has an important role in the demonstration of the dehiscence of the SSC confirming the diagnosis of SSC dehiscence syndrome. ${ }^{9,10}$ However, temporal bone CT accuracy depends on the availability of multidetector row CT capable of thin-section collimation with isotropic voxel allowing multiplanar reformation of the images. ${ }^{11-14}$

\footnotetext{
${ }^{1}$ DIBMEF-Sezione di Scienze Radiologiche, Università degli Studi di Palermo, Italy

${ }^{2}$ Neuroradiology Section, Christiana Care Health System, USA
}

\section{Corresponding author:}

Gianvincenzo Sparacia, DIBIMEF-Sezione di Scienze Radiologiche, Università degli Studi di Palermo, Via Del Vespro, 127, 90127, Palermo, Italy.

Email: gianvincenzo.sparacia@unipa.it 
Previous studies reported the sensitivity and specificity of CT imaging using $1-\mathrm{mm}$ collimation or $0.5-\mathrm{mm}$ collimation in the detection of SSC dehiscence. ${ }^{9,12-14}$

However, significant differences have been found between autopsy-proven versus CT-detected SSC dehiscence. ${ }^{15-17}$ It has been proposed that reformatted CT images oriented parallel (plane of Pöschl) and orthogonal (plane of Stenver) to the plane of the SSC may improve the sensitivity and specificity in detecting SSC dehiscence. ${ }^{10-13}$

The aim of our study was to establish the diagnostic performance of $0.5-\mathrm{mm}$ collimation temporal bone helical CT in the diagnosis of SSC dehiscence syndrome comparing the accuracy of reformatted images oriented in the standard axial and coronal planes, as compared with the accuracy of reformatted images oriented parallel and perpendicular to the SSC.

This information is fundamental for the radiologic diagnosis of this entity, particularly in cases where surgical correction of the abnormality is considered.

\section{Material and methods}

\section{Patient population}

The institutional review board of University of Palermo, Italy approved this retrospective study and written informed consent was obtained from all patients.

From January 2015 through June 2016, 42 patients ( 25 men, 17 women, mean age 46 years, range $22-75$ years) affected by sound- and/or pressure-induced vestibular symptoms underwent clinical evaluation that included monitoring of sound- and pressure-evoked eye movements.

In all patients, SSC dehiscence syndrome was established based on the evidence of vertical-torsional eye movements in the plane of the SSC evoked by sound and/or pressure stimuli as previously described. ${ }^{12}$

Forty-two age- and sex-matched individuals (25 men, 17 women, mean age 48 years, range 19-86 years), who underwent multidetector row CT examination in the same period for temporal bone disorders, in the absence of sound- or pressure-induced symptoms and without bone erosions at CT examination, were randomly selected as a control group.

A total of $84 \mathrm{CT}$ examinations, covering the bilateral temporal bones of each patient and control individuals, were considered, allowing the evaluation of 168 ears (84 left and 84 right ears) and their corresponding SSCs.

\section{CT scans}

All patients and controls underwent helical $0.5-\mathrm{mm}$ collimated temporal bone CT. Thin-section CT scans were obtained in the axial plane with a 64-row CT scanner (Brilliance CT, Philips, Best, The Netherlands) with the following parameters: tube voltage $=120 \mathrm{kV}$, effective $\mathrm{mAs}=330$, slice thickness $0.55 \mathrm{~mm}$, spacing $0.3 \mathrm{~mm}$, pitch $=0.7$, field of view $(\mathrm{FOV})=180 \mathrm{~mm}$, image matrix $768 \times 768$.

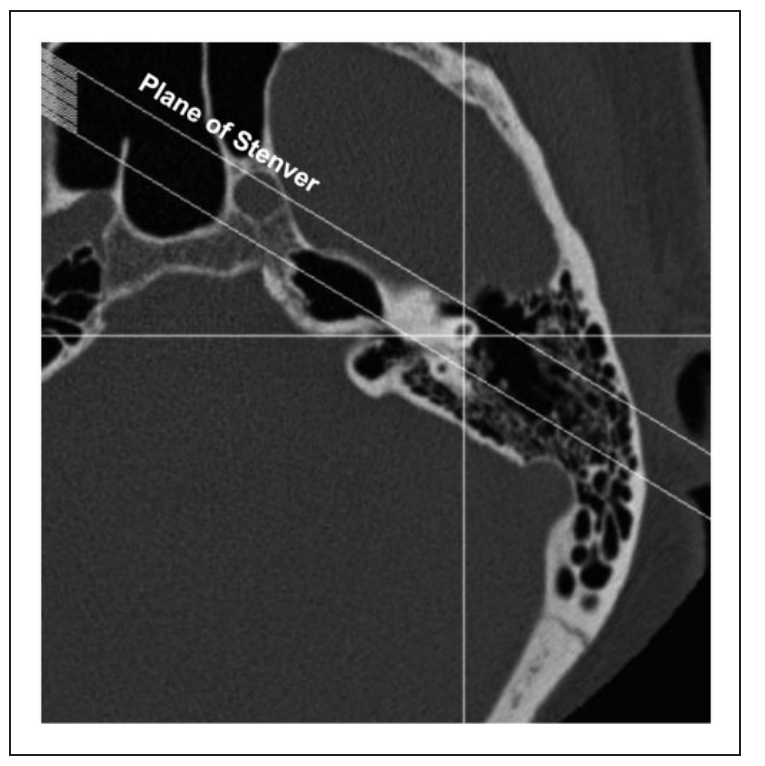

Figure 1. Plane of Stenver. Orientation of the plane of reformation perpendicular to the superior semicircular canal demonstrated on the axial scout image.

A volume between 3.5 and $4.5 \mathrm{~cm}$ was acquired through the temporal bone.

The acquisition time was 30 seconds. The raw data were reconstructed by using the bone algorithm.

Reformatted images of the left and right temporal bones of patients and controls were obtained from this volumetric isotropic dataset for the purpose of this study by using a dedicated workstation (Philips, Best, The Netherlands).

Standard axial and coronal images through the temporal bone were reconstructed at $0.1-\mathrm{mm}$ increments.

Fifteen oblique reformatted images, perpendicular to the SSC (plane of Stenver), were obtained with a space gap of $0.1 \mathrm{~mm}$ (Figure 1).

Fifteen oblique reformatted images, parallel to the SSC (plane of Pöschl) (Figure 2), were obtained with a space gap of $0.1 \mathrm{~mm}$ to allow visualization of the SSC in its sagittal plane (Figure 3).

\section{Image evaluation}

The images were presented in a random order on a picture archiving and communication system (PACS) (Agfa HealthCare $\mathrm{GmbH}$, Bonn, Germany) by two neuroradiologists, each with at least 10 years' experience.

Dehiscence of the SSC was defined by the absence of high-attenuation bone coverage on the $\mathrm{CT}$ images.

Images were scored on the basis of the integrity of the SSC as no dehiscence present or dehiscence present. Any discrepancies between the two neuroradiologists were resolved by consensus.

Initially, the standard axial and coronal images (group A images) of each temporal bone (left or right ear) were evaluated. 


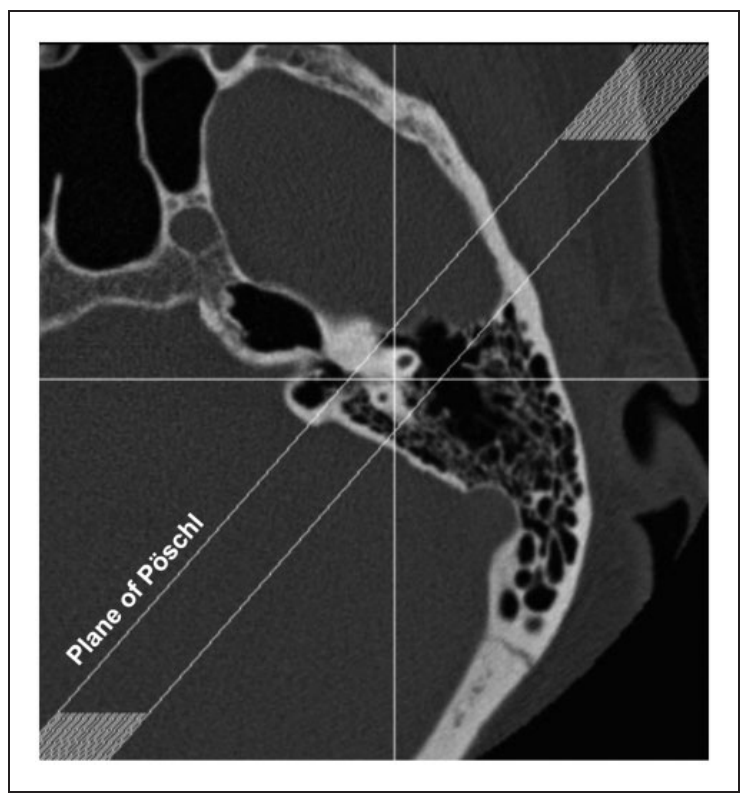

Figure 2. Plane of Pöschl. Orientation of the plane of reformation parallel to the superior semicircular canal demonstrated on the axial scout image.

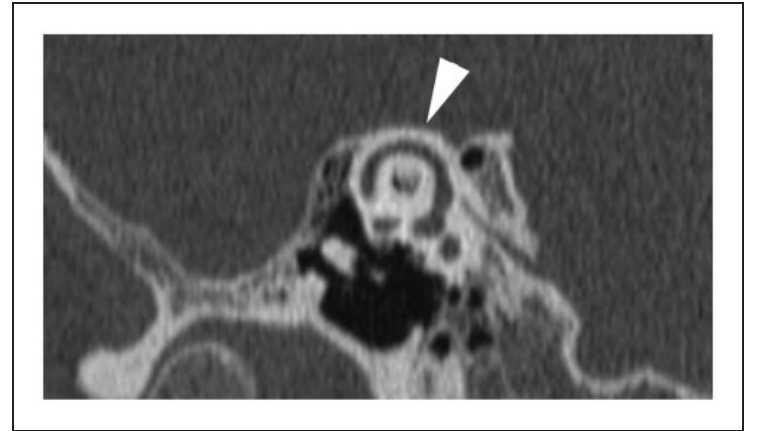

Figure 3. Thin-section reformatted computed tomography image oriented in the plane of Pöschl demonstrating an intact superior semicircular canal (arrowhead) visualized entirely in its sagittal plane.

Subsequently, the reformatted images oriented in parallel (plane of Pöschl) and perpendicular (plane of Stenver) planes to the SSC (group B images) were evaluated for each temporal bone (left or right ear).

True-positive (TP) results, for group A or group B images, were those that demonstrated dehiscence of the SSC in a patient with a positive clinical test (observation of the typical eye movements in the plane of the affected SSC evoked by sound or pressure stimuli).

False-negative $(\mathrm{FN})$ results, for group A or group B images, were those with a positive clinical test and that demonstrated no SSC dehiscence.

\section{Statistical analysis}

To compare the diagnostic performance obtained evaluating group $\mathrm{A}$ images and group B images, the area under the receiver operating characteristic (ROC) curve (AUC) analysis was used.

The Hanley and McNeil method ${ }^{18}$ was used for comparing the two AUCs with the reminder that a larger AUC suggests better performance of a test.

Differences were considered statistically significant at $p<0.05$.

Sensitivity, specificity, TP, true negative (TN), false positive (FP), FN as well as the accuracy of the results obtained evaluating group A and group B images were calculated.

Statistical software used was Analyse-It (Analyse-it Software, Ltd, Leeds, United Kingdom) for Microsoft Excel (Microsoft Corporation, Redmond, WA, USA).

\section{Results}

The diagnosis of SSC dehiscence syndrome was established clinically on the basis of the characteristic symptoms and evidence of vertical-torsional eye movements in the plane of the SSC evoked by sound and/or pressure stimuli. There were 25 left ears solely affected and 17 right ears solely affected in the 42 patients. There were no cases of bilateral involvement of SSC dehiscence syndrome.

On CT, SSC dehiscence was defined by the absence of high-attenuation bone coverage on the reformatted images.

Diagnostic performance results for group A and group B images evaluation are presented in Table 1.

Group A images evaluation demonstrated an AUC of 0.929 (sensitivity $85.7 \%$, specificity $100 \%$, TP 36, TN 42 , FP 0, FN 6) with an overall accuracy of $92.9 \%$.

Figure 4 shows a representative case of SSC dehiscence, demonstrated on the reformatted standard coronal image.

Group B images evaluation demonstrated an AUC of 0.988 (sensitivity $97.6 \%$, specificity $100 \%$, TP 41 , TN 42 , FP 0, FN 1) with an overall accuracy of 98.8 .

Representative cases of SSC dehiscence demonstrated on the reformatted images oriented in the plane of Stenver and in the plane of Pöschl are presented in Figure 4 and 5, respectively.

Although the differences between the two AUCs were not statistically significant, evaluation of group $\mathrm{B}$ images alone showed an improved diagnostic performance over group A images alone.

In particular, for group A images, SSC dehiscence was correctly demonstrated in 36 out of 42 temporal bones, with six FN cases, whereas for group B images, SSC dehiscence was correctly demonstrated in 41 out of 42 temporal bones, with only one FN case.

\section{Discussion}

In our study, the accuracy of thin-section $(0.5 \mathrm{~mm})$ helical CT for the detection of dehiscence of the SSC was improved by using images reformatted in planes parallel (plane of Pöschl) and perpendicular (plane of 
Table 1. Diagnostic performance results for group A images and group B images in the detection of superior semicircular canal (SSC) dehiscence in 42 patients clinically diagnosed with SSC dehiscence syndrome and in 42 control participants.

\begin{tabular}{|c|c|c|c|c|c|c|c|c|}
\hline & Sensitivity & Specificity & $A \cup C$ & Accuracy & TP & TN & FP & $\mathrm{FN}$ \\
\hline Group A images & $85.7 \%$ & $100.0 \%$ & 0.929 & $92.9 \%$ & 36 & 42 & 0 & 6 \\
\hline Group B images & $97.6 \%$ & $100.0 \%$ & 0.988 & $98.8 \%$ & 41 & 42 & 0 & 1 \\
\hline
\end{tabular}

Group A images: reformatted CT images of the temporal bone oriented in the standard transverse and coronal planes. Group B images: reformatted CT images of the temporal bone oriented in parallel (plane of Pöschl) and perpendicular (plane of Stenver) planes to the SSC. AUC: area under the receiver operating characteristic curve; TP: true positive, TN: true negative, FP: false positive, FN: false negative; CT: computed tomography.

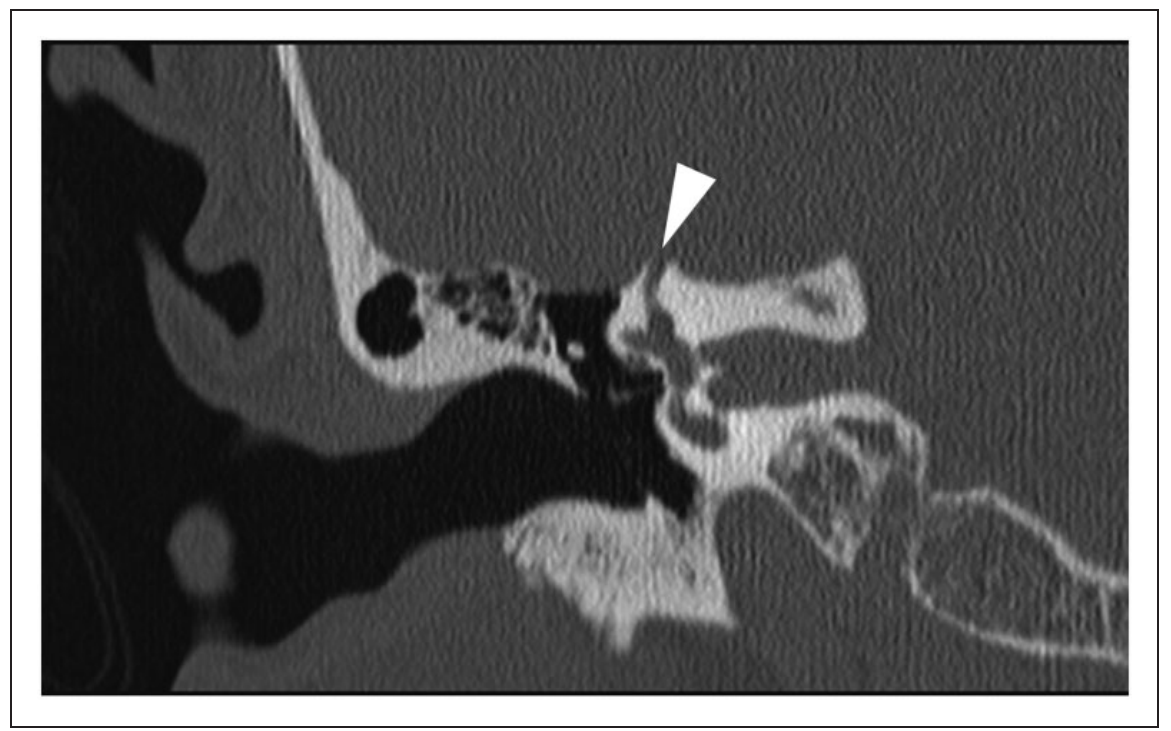

Figure 4. Thin-section reformatted computed tomography image oriented in the standard coronal plane demonstrating the dehiscence of the superior semicircular canal (arrowhead).

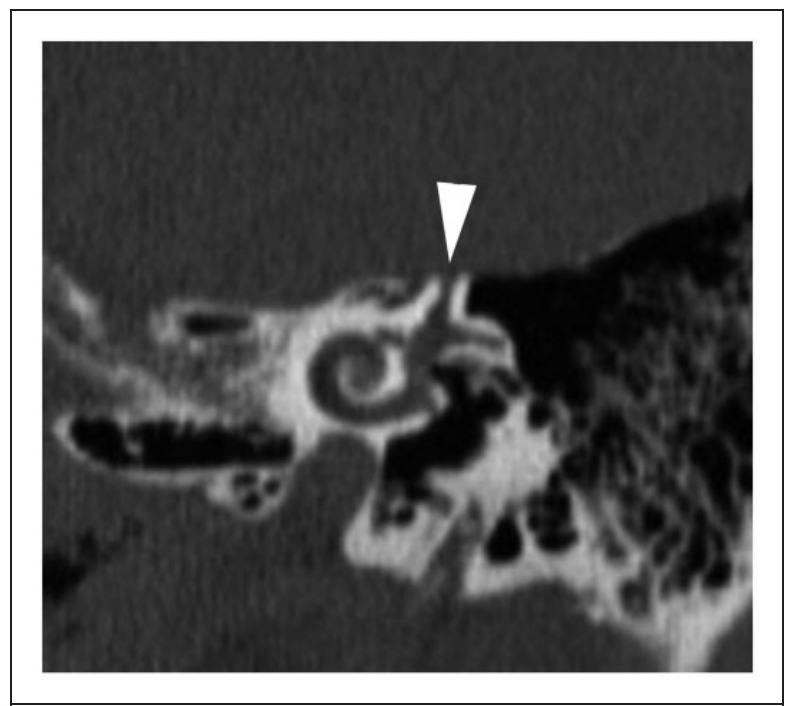

Figure 5. Thin-section reformatted computed tomography image oriented in the plane of Stenver demonstrating the dehiscence of the superior semicircular canal (arrowhead).

Stenver) to the SSC (Figures 5 and 6). In a previous study, Branstetter et al. ${ }^{13}$ suggested that these additional reformatted images are not routinely needed to diagnose SSC dehiscence, indicating that they should be

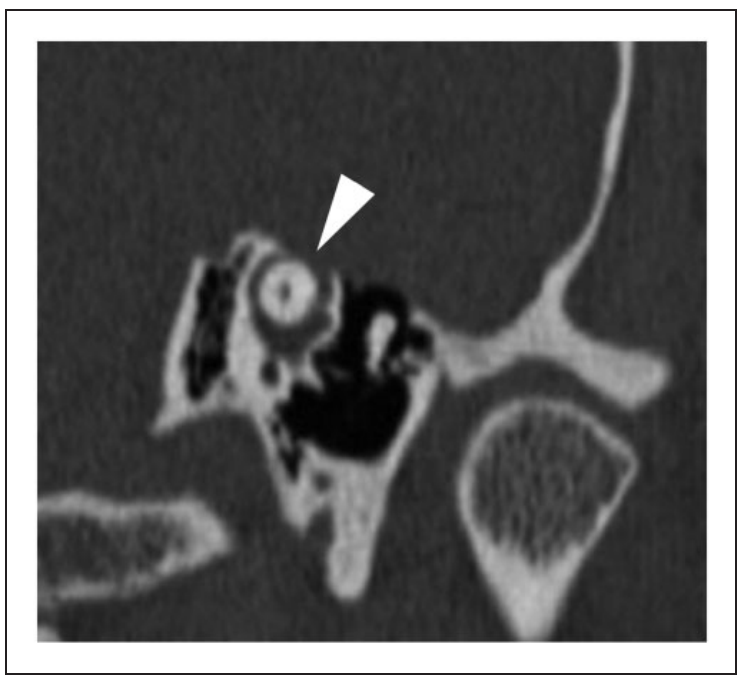

Figure 6. Thin-section reformatted computed tomography (CT) image oriented in the plane of Pöschl demonstrating the dehiscence of the superior semicircular canal (arrowhead).

performed only when the diagnosis remains uncertain after review of the standard coronal reformatted images.

However, Branstetter et al. ${ }^{13}$ acknowledged as a limitation of their study their failure to include an 
"oblique reformation only" group, an aspect that is indeed addressed by our study.

Moreover, our results demonstrate that these additional reformatted images alone, oriented in the parallel and perpendicular plane to the SSC, provide an accuracy of $98.8 \%$ compared to the accuracy of $92.9 \%$ of the standard axial and coronal reformatted images alone. Thus, these additional reformatted images alone provide an improvement of the accuracy in the diagnosis of SSC dehiscence syndrome, which results in six missed cases by evaluating the group A images compared to the only one missed case by evaluating the group B images.

The explanation of the reduced diagnostic performance of group A images relies on the well-known phenomenon of partial volume averaging, which affects mainly those images in which the roof of the SSC is oblique to the coronal plane of the section and therefore fills only partially the voxels that compose the image. ${ }^{12}$ Conversely, the effect of partial volume averaging can be reduced by using a parallel plane to the SSC for the visualization of the roof of the SSC, thus allowing virtually complete filling of the voxels that comprise the image.

Although these additional reformatted images require more time for the post-processing, no additional radiation or scanning time are necessary and the benefit in terms of improvement in the accuracy for the diagnosis is clearly demonstrated.

While SSC dehiscence syndrome can be diagnosed on clinical grounds, CT imaging is required to confirm the diagnosis and to localize the portion of the SSC that is dehiscent; this aspect of the diagnosis is particularly critical to confirm whenever surgical treatment is contemplated. $^{12,19,20}$

The surgical treatment consists in surgically obliterating the defect in the superior canal by using either a trans-mastoid or middle fossa approach. ${ }^{17,21}$

Previous studies suggest that radiologic evidence of SSC dehiscence may be overestimated, indicating that a dehiscence at the arcuate eminence may be observed in asymptomatic patients. ${ }^{14,16,17}$

It should be pointed out, however, that these studies are based on older scanning protocols with 1.0-mmthick direct transverse and coronal images, or with $1.0-\mathrm{mm}$-thick reformatted images in the plane of Pöschl and Stenver.

Other studies, based on 0.5-mm collimation CT scan with orthogonal reformatted images, reported a higher prevalence of SSC dehiscence in comparison to the prevalence reported in a post-mortem study of 1000 temporal bones, confirming that advanced, thin-section, multirow CT scans improve the diagnostic accuracy of the technique in the detection of the SSC dehiscence. $^{19}$

Our results confirm that multislice temporal bone CT with $0.5-\mathrm{mm}$ collimation and reformatted images to the planes of Pöschl and Stenver improves the specificity and the positive predictive value of the technique as reported in the literature. ${ }^{12,13}$ Moreover, this study provides evidence that temporal bone CT with $0.5-\mathrm{mm}$ collimation and reformatted images in the plane of Pöschl and Stenver alone provides the best overall diagnostic performance (accuracy 98.8\%).

This study, as previous studies in the literature, ${ }^{9,12-15,19}$ is limited by the lack of surgical confirmation of the CT diagnosis but, clearly, patients with negative radiologic findings are not likely to be considered for surgical exploration and, similarly, not all patients with a radiologic diagnosis and clinical evidence of SSC dehiscence choose to undergo surgery.

It was proposed that multidetector row $\mathrm{CT}$ imaging of SSC dehiscence could be superseded by digital volume tomography (DVT), also known cone beam computed tomography (CBCT), which provides fast image acquisition with relatively high three-dimensional resolution, and lower radiation exposure compared with multidetector row $\mathrm{CT} .{ }^{22-25}$

However, it should be mentioned that not all image data sets for DVT from different companies are equally accurate, and that the majority of the studies in the literature are based on in vitro examinations, ${ }^{24,25}$ thus, further studies are needed to validate DVT in the detection of SSC dehiscence in a large sample population.

In conclusion, our results provide evidence that proper CT examination with $0.5-\mathrm{mm}$ collimation and reformatted images into the Pöschl and Stenver planes improves detection of potentially treatable SSC dehiscence in patients with vestibular symptoms induced by sound and/or pressure stimuli.

DVT has the potential to be an excellent alternative to multidetector row CT for inner ear imaging with lower radiation to patients and equal or higher resolution depending on the unit used.

\section{Funding}

This research received no specific grant from any funding agency in the public, commercial, or not-for-profit sectors.

\section{Conflict of interest}

The authors declared no potential conflicts of interest with respect to the research, authorship, and/or publication of this article.

\section{References}

1. Minor LB, Solomon D, Zinreich JS, et al. Sound- and/or pressure-induced vertigo due to bone dehiscence of the superior semicircular canal. Arch Otolaryngol Head Neck Surg 1998; 124: 249-258.

2. Tullio P. Das Ohr und die Entstehung der Sprache und Schrift. Berlin, Germany: Urban and Schwarzenber, 1929.

3. Nadol JB Jr. Positive Hennebert's sign in Meniere's disease. Arch Otolaryngol 1977; 103: 524-530.

4. Minor LB. Superior canal dehiscence syndrome. Am J Otol 2000; 21: 9-19.

5. Minor LB. Clinical manifestations of superior semicircular canal dehiscence. Laryngoscope 2005; 115: 1717-1727. 
6. Ostrowski VB, Byskosh A and Hain TC. Tullio phenomenon with dehiscence of the superior semicircular canal. Otol Neurotol 2001; 22: 61-65.

7. Basura GJ, Cronin SJ and Heidenreich KD. Tullio phenomenon in superior semicircular canal dehiscence syndrome. Neurology 2014; 82: 1010.

8. Lee JJ, Ohorodnyk P, Sharma M, et al. Bilateral superior semicircular canal dehiscence and Tullio phenomenon. Can J Neurol Sci 2016; 43: 319-321.

9. Sequeira SM, Whiting BR, Shimony JS, et al. Accuracy of computed tomography detection of superior canal dehiscence. Otol Neurotol 2011; 32: 1500-1505.

10. Mong A, Loevner LA, Solomon D, et al. Sound- and pressure-induced vertigo associated with dehiscence of the roof of the superior semicircular canal. AJNR Am J Neuroradiol 1999; 20: 1973-1975.

11. Curtin HD. Superior semicircular canal dehiscence syndrome and multi-detector row CT. Radiology 2003; 226: 312-314.

12. Belden CJ, Weg N, Minor LB, et al. CT evaluation of bone dehiscence of the superior semicircular canal as a cause of sound- and/or pressure-induced vertigo. Radiology 2003; 226: 337-343.

13. Branstetter BF, 4th, Harrigal C, Escott EJ, et al. Superior semicircular canal dehiscence: Oblique reformatted CT images for diagnosis. Radiology 2006; 238: 938-942.

14. Ceylan N, Bayraktaroglu S, Alper H, et al. CT imaging of superior semicircular canal dehiscence: Added value of reformatted images. Acta Otolaryngol 2010; 130: 996-1001.

15. Yew A, Zarinkhou G, Spasic M, et al. Characteristics and management of superior semicircular canal dehiscence. J Neurol Surg B Skull Base 2012; 73: 365-370.

16. Williamson RA, Vrabec JT, Coker NJ, et al. Coronal computed tomography prevalence of superior semicircular canal dehiscence. Otolaryngol Head Neck Surg 2003; 129: 481-489.
17. Stimmer H, Hamann KF, Zeiter S, et al. Semicircular canal dehiscence in HR multislice computed tomography: Distribution, frequency, and clinical relevance. Eur Arch Otorhinolaryngol 2012; 269: 475-480.

18. Hanley JA and McNeil BJ. A method of comparing the areas under receiver operating characteristic curves derived from the same cases. Radiology 1983; 148: 839-843.

19. Inal M, Burulday V, Bayar Muluk N, et al. Magnetic resonance imaging and computed tomography for diagnosing semicircular canal dehiscence. $J$ Craniomaxillofac Surg 2016; 44: 998-1002.

20. Brantberg K, Bergenius J, Mendel L, et al. Symptoms, findings and treatment in patients with dehiscence of the superior semicircular canal. Acta Otolaryngol 2001; 121: 68-75.

21. Huizinga E. On the sound reaction of Tullio in the pigeon and the fenestration operation in man. Acta Otolaryngol Suppl 1952; 100: 174-180.

22. Sepúlveda I, Schmidt T and Platín E. Use of cone beam computed tomography in the diagnosis of superior semicircular canal dehiscence. J Clin Imaging Sci 2014; 4: 49.

23. Dalchow CV, Knecht R, Grzyska U, et al. Radiographic examination of patients with dehiscence of semicircular canals with digital volume tomography. Eur Arch Otorhinolaryngol 2013; 270: 511-519.

24. Bremke M, Luers JC, Anagiotos A, et al. Comparison of digital volume tomography and high-resolution computed tomography in detecting superior semicircular canal dehiscence - a temporal bone study. Acta Otolaryngol 2015; 135: 901-906.

25. Teymoortash A, Hamzei S, Murthum T, et al. Temporal bone imaging using digital volume tomography and computed tomography: a comparative cadaveric radiological study. Surg Radiol Anat 2011; 33: 123-128. 